\title{
Protective Effects of ACEI/ARB on Left Ventricular Function in Anthracycline-Induced Chronic Cardiotoxicity: A Meta-Analysis of Randomized Controlled Trials
}

\author{
Hong Lin ${ }^{a, b}$ Guoxi Lianga, ${ }^{a}$ Yanxuan Wu ${ }^{b, c}$ Liming Chen ${ }^{a}$ \\ aDepartment of Oncology, The First Affiliated Hospital of Shantou University Medical College, Shantou, China; \\ bShantou University Medical College, Shantou, China; 'Department of Radiation Oncology, Cancer Hospital of \\ Shantou University Medical College, Shantou, China
}

\section{Keywords}

Anthracyclines · Angiotensin-converting enzyme inhibitors . Angiotensin-receptor antagonists · Cardiotoxicity .

Meta-analysis

\begin{abstract}
Purpose: Cardiotoxicity is an important side effect of anthracycline. Cardioprotective drugs for anthracycline remain inconclusive. We attempted to determine the role of angiotensin-converting enzyme inhibitors (ACEI) and angiotensin-receptor blockers (ARB) in the prevention of anthracycline-induced cardiotoxicity. Hypothesis: Prophylactic use of ACEI/ARB reduces the clinical or subclinical cardiotoxicity of anthracycline. Methods: Randomized controlled trials (RCTs) of ACEI/ARB in the prevention of anthracyclineinduced cardiotoxicity were obtained by searching Pubmed, Embase, Web of Science, and Cochrane databases. 7 studies were finally included. A meta-analysis was performed on the 7 studies. The end points were changes in left ventricle ejection fraction (LVEF), early and late diastolic peak velocity ratio $(E / A)$, and occurrence of hypotensive events. Results: Prophylactic use of ACEI/ARB has potential benefits for anthracycline-induced cardiotoxicity. LVEF was better preserved in the experimental group than in the control group (weighted mean difference [WMD] $-3.16 \%, 95 \%$ confidence interval
\end{abstract}

$[\mathrm{Cl}][-5.78,-0.54], p=0.02)$. Follow-up time, tumor type, drug type, and geographical region did not affect the results. There was no significant benefit of $E / A$ in the experimental group (WMD $0.02,95 \% \mathrm{Cl}[-0.06,0.11], p=0.58$ ), and no increase in the incidence of hypotension (risk ratio $3.79,95 \%$ $\mathrm{Cl}[0.44,32.89], p=0.23)$. Conclusions: We found that prophylactic use of ACEI/ARB reduced the clinical or subclinical cardiotoxicity of anthracycline, and the increase in hypotensive events was not significant. Due to the relatively small number of clinical studies and participants, more related studies are necessary to further verify our results.

(c) 2021 S. Karger AG, Base

\section{Introduction}

Cancer is the second leading disease in the world after cardiovascular disease. Current cancer treatments include surgery, chemotherapy, radiotherapy, targeted therapy, biotherapy, and immunotherapy. Anthracycline chemotherapeutic agents are important drugs to treat cancer. Chemotherapy regimens containing anthracycline are widely used in the treatment of hematologic malignancies, breast cancer, soft tissue sarcoma, and other malignancies. Dose-dependent cardiotoxicity is one of the important side effects of anthracycline. With the im- karger@karger.com

www.karger.com/crd
Karger"

(c) 2021 S. Karger AG, Base
Correspondence to:

Liming Chen, angelchen09@163.com 
Fig. 1. Flowchart of trials selection process. RCT, randomized controlled trial; ACEI, angiotensin-converting enzyme inhibitors; $\mathrm{ARB}$, angiotensin-receptor blockers

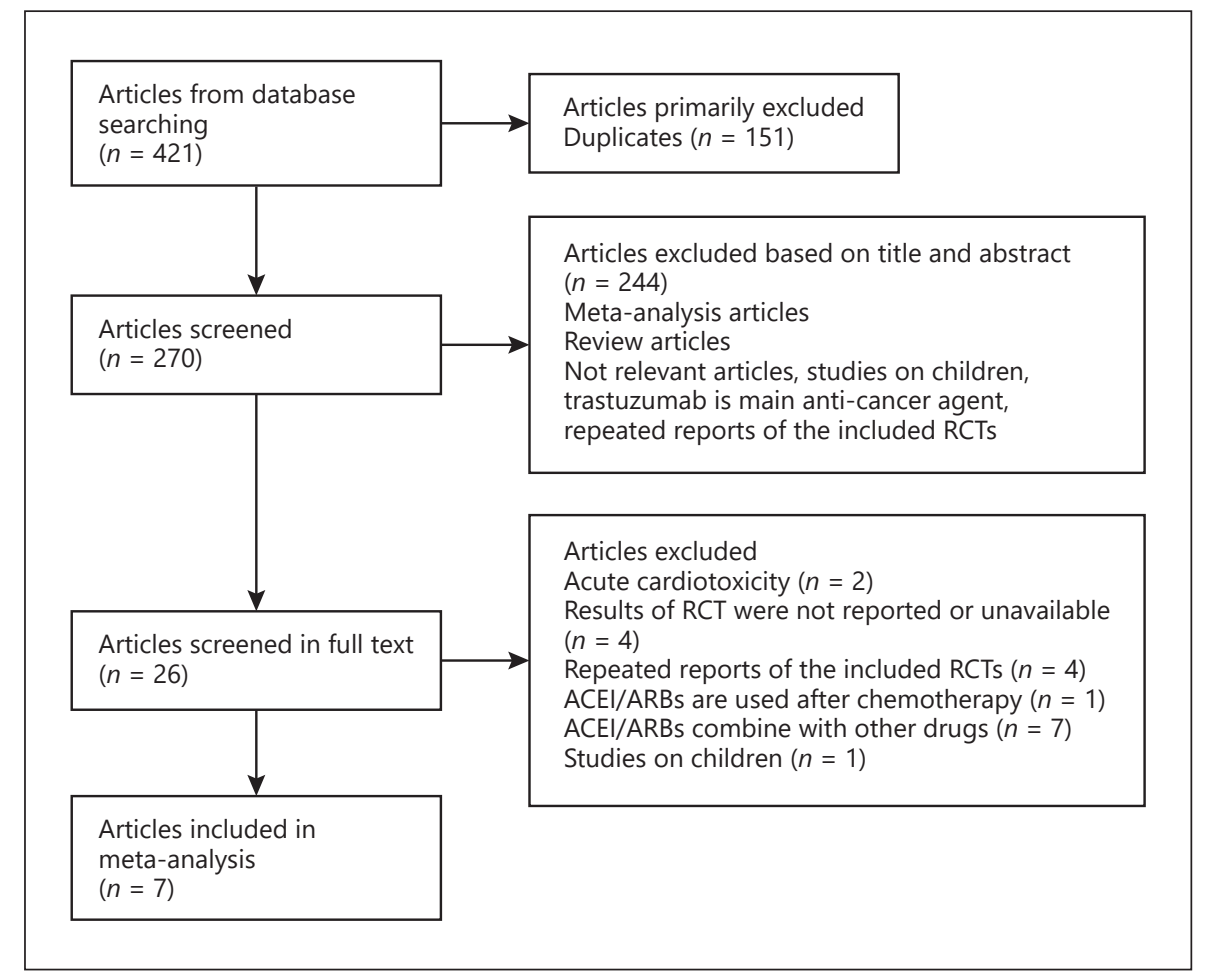

provement of cancer treatment methods, the survival time of cancer patients is gradually prolonging, and the long-term effects of anthracycline on heart function are gradually attracting people's attention. Clinical guideline [1] issued by the American Society for Clinical Oncology recommends cardioprotective measures for patients using the following treatment: (1) using high-dose anthracycline (such as doxorubicin $>250 \mathrm{mg} / \mathrm{m}^{2}$ or epirubicin $>600 \mathrm{mg} / \mathrm{m}^{2}$ ); (2) low-dose anthracycline (such as doxorubicin $<250 \mathrm{mg} / \mathrm{m}^{2}$ or epirubicin $<600 \mathrm{mg} / \mathrm{m}^{2}$ ) with trastuzumab to targeted therapy or radiotherapy; and (3) patients more than 60 years old, or with more than one existing cardiovascular risk factor, or known cardiovascular disease. However, only dexrazoxane is currently recommended for the prevention of cardiotoxicity. Other agents, such as angiotensin-converting enzyme inhibitor (ACEI), angiotensin-receptor blocker (ARB), and adrenergic beta-receptor blocker, are not recommended for first-line use due to the lack of sufficient evidence.

As a renin-angiotensin-aldosterone system inhibitor, ACEI/ARB can inhibit ventricular remodeling to improve the prognosis of patients with heart failure, and its protective effect on the heart has been proven. In the last 20 years, some studies have begun to explore whether ACEI/ARB can also protect the heart from cardiotoxicity induced by anthracycline, and the results were inconsis- tent. The evaluation of left ventricular ejection fraction (LVEF) is widely used in various studies because it is the most important reference indicator for changes in cardiac function at present. This meta-analysis aims to determine the effect of the prophylactic use of ACEI/ARB on the clinical and subclinical cardiotoxicity induced by anthracycline.

\section{Methods}

This meta-analysis was performed in accordance with the PRISMA [2] and the Cochrane Handbook guidelines [3]. The PRISMA 2009 checklist is provided (see online suppl. material at www.karger.com/doi/10.1159/000512848).

\section{Database Search}

We search articles on Pubmed, Embase, Cochrane, and Web of Science databases (until February Week 3, 2020). We used the following terms to search: "anthracycline" or "epirubicin" or "doxorubicin" and one of "ACEI," "ARB," "Angiotensin-Converting Enzyme Inhibitors," "Angiotensin Receptor Antagonists." Only articles published in English were considered. Two reviewers (Lin and Liang) screened the articles independently.

\section{Study Selection}

Studies which met the following criteria were included: (1) only clinical randomized controlled trial (RCT) that evaluated the use of ACEI/ARB in the prevention of cardiotoxicity in patients re- 


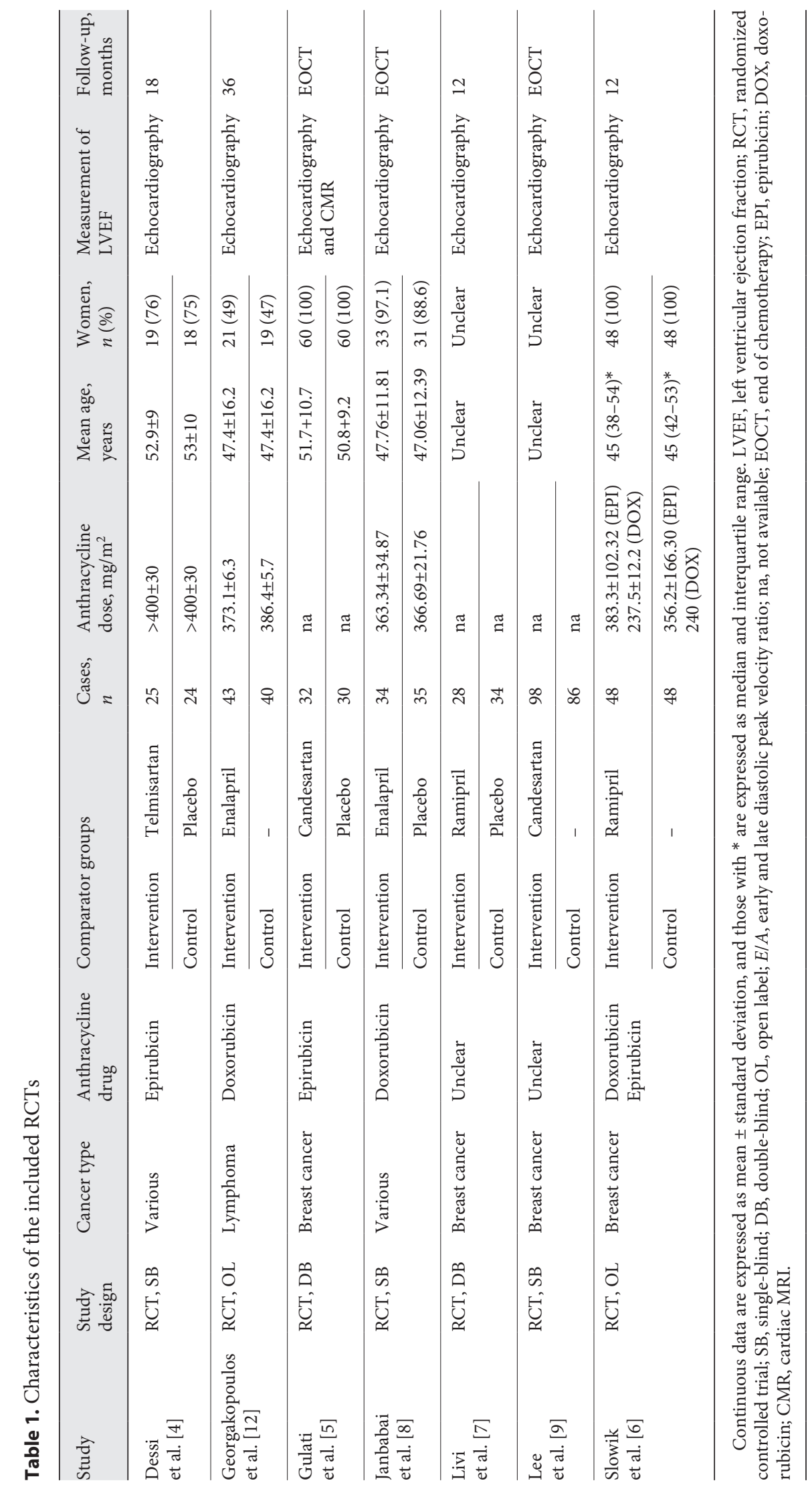


Table 2. Extracted data from studies

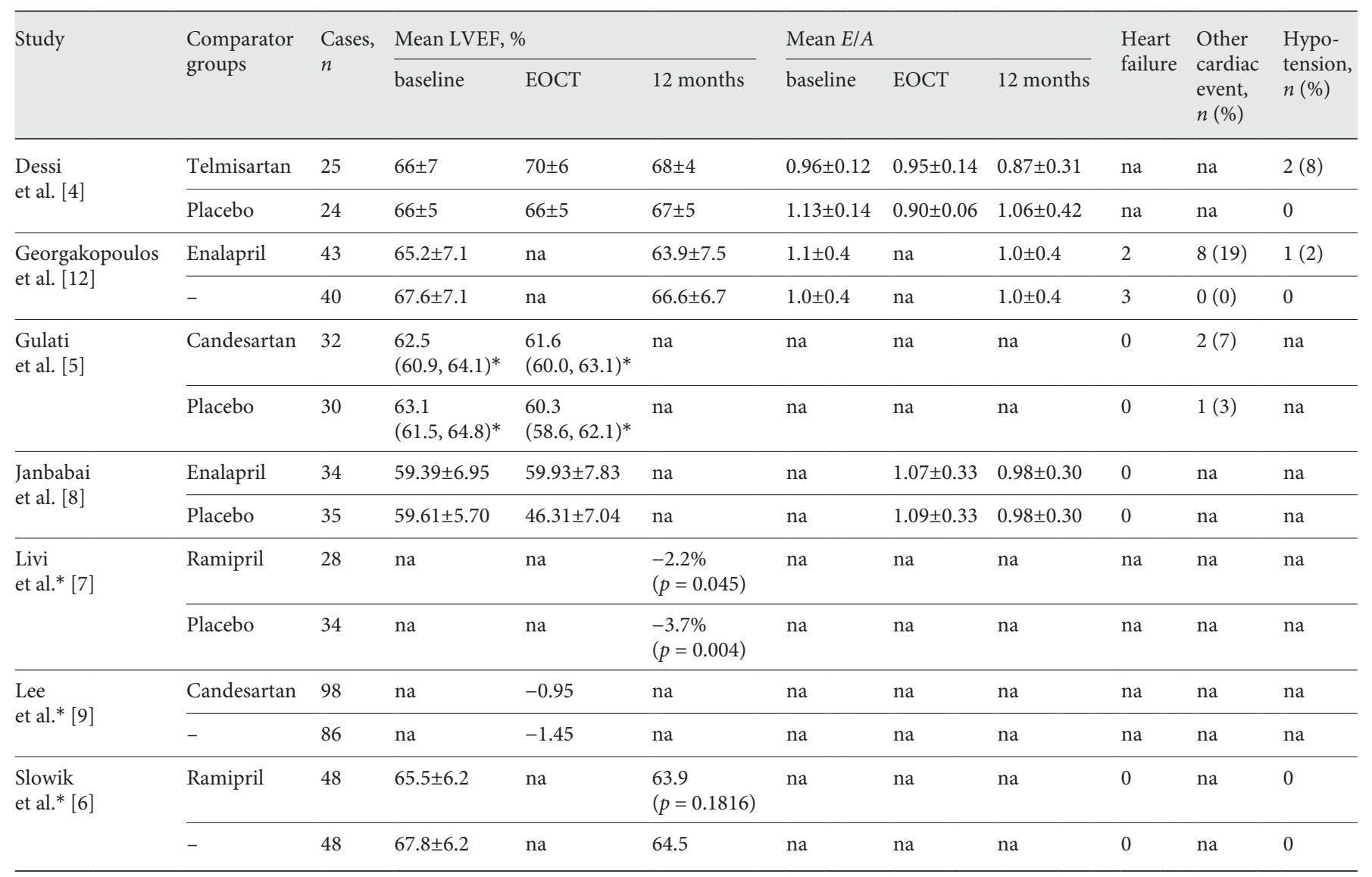

LVEF, left ventricular ejection fraction; E/A, early and late diastolic peak velocity ratio; na, not available; EOCT, end of chemotherapy. Continuous data are expressed as mean \pm standard deviation. * Studies offered mean or mean difference of LVEF.

ceiving anthracycline-based chemotherapy was included; (2) included participants in the trial were adults ( $>18$ years old); (3) LVEF is a primary indicator of cardiotoxicity and is obtained by cardiac MRI (CMR) or echocardiography; (4) ACEI/ARB was used before or in conjunction with anthracycline; and (5) results of RCT were published or available. Trials for the study of acute cardiotoxicity of anthracycline were not included. Trials involving ACEI/ $\mathrm{ARB}$ in combination with other drugs, such as beta-receptor blockers or ACEI/ARB as controls were also excluded.

\section{Data Extraction and Quality Assessment}

Two reviewers (Lin and Liang) extracted data, respectively, and performed the quality assessment. A consensus was negotiated if there was a discrepancy. We used the Cochrane Collaboration's tool to evaluate the risk of bias. Collected data included the year of publication, nationality, study design characteristics, cancer type, anthracycline agent and dose, intervention, characteristics of patients, follow-up time, method for the measurement of LVEF, data on LVEF, data on early and late diastolic peak velocity ratio $(E / A)$ (pre-anthracycline and post-anthracycline), cardiac events, and hypotensive events.

\section{End Points and Statistics}

The primary end point of this meta-analysis was the mean difference of LVEF changes between the ACEI/ARB and control groups after anthracycline-based chemotherapy. It was calculated by subtracting the baseline mean from the post-anthracycline mean. We also performed subgroup analyses on follow-up time, drug type, tumor type, and geography to evaluate whether the potential benefit of ACEI/ARB for the preservation of LVEF was influenced by the above factors. The secondary outcome was $E / A$, which represented the diastolic function of the left ventricular. Safety outcome was the incidence of hypotension. For continuous data synthesis, mean and standard deviation were recorded. If data were not provided directly, we used the Revman calculator to calculate. The final results were expressed as weighted mean difference (WMD). As for dichotomous data, it was analyzed using risk ratios. Both 2 kinds of data calculated 95\% confidence interval (CI). We used the Cochran Q test and the $I^{2}$ statistic to assess heterogeneity. A random-effects model was used if there was substantial heterogeneity ( $p$ value $<0.05$ in $Q$ test or $I^{2}>50 \%$ ). Otherwise, a fixed-effects model was used. The potential publication bias is visually checked by a funnel plot. We did not perform Egger's visual inspection test because there were less than 10 studies. We conducted a post hoc sensitivity analysis for the 


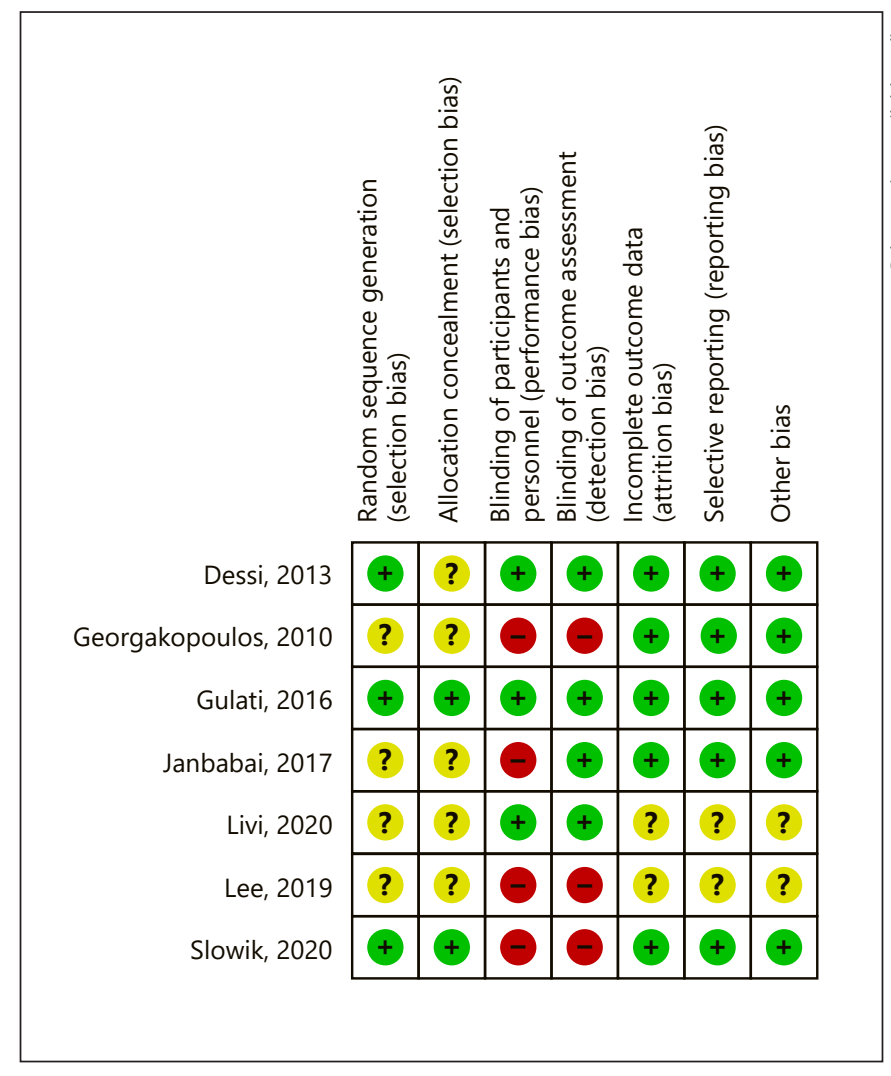

Fig. 2. Risk of bias summary of 7 RCTs. RCT, randomized controlled trial.

considerable heterogeneity. One of the RCTs had markedly different results (see below), so we excluded it and performed meta-analysis again. $p$ values $<0.05$ were considered statistically significant. Statistical study was performed by using Revman software (Version 5.3, Cochrane, Oxford, UK).

\section{Result}

\section{Search Result and Patients}

A PRISMA flow diagram is shown in Figure 1. A total of 7 RCTs were enrolled with 605 patients (308 in the ACEI/ARB group and 297 in the control group). Of these studies, 5 RCTs have full-text publications, while the other 2 are conference abstracts. There are 4 RCTs for breast cancer and 3 for various cancers including lymphoma, leukemia, breast cancer, and so on. The major anthracyclines used are doxorubicin and epirubicin. The baseline values of LVEF were reported in 5 studies. Four studies reported LVEF related values post-anthracycline. Four studies reported LVEF related values after a 12-month follow-up duration. All of the LVEF values were obtained using echocardiography. One of which was also per-

ACEI/ARB Reduces Anthracycline-

Induced Cardiotoxicity formed with cardiac MRI. Three studies reported E/A. Four studies documented heart failure events. Three studies reported the occurrence of hypotensive events.

Table 1 shows the characteristics of these 7 studies. Table 2 shows the data extracted from each study.

\section{Quality Evaluation}

The bias assessment of involving RCTs is presented in Figure 2. There were 3 studies [4-6] described the method of random sequence generation clearly, of which 2 studies $[5,6]$ also described allocation concealment. Three studies $[4,5,7]$ described a rigorous blinding method for participants, while 4 RCTs $[4,5,7,8]$ used standard blinding method to assess the outcomes of studies. Except for 2 conference abstracts that were not explicitly reported [7, 9], other 5 studies were evaluated as having a low risk of attrition bias and reporting bias.

\section{Result of the Primary End Point}

Due to the large heterogeneity, a random-effects model was used to evaluate the mean difference of LVEF change ( $p$ value $<0.00001$ in $Q$ test and $I^{2}>50 \%$ ). The pooled result is shown in Figure 3a. Compared with the control group, patients treated with ACEI/ARB preserved LVEF better although the improvement of LVEF by ACEI/ARB was small (WMD -3.16\%, 95\% CI [-5.78, -0.54], $p=0.02$ ). Subgroup analyses show no significant difference in follow-up duration, tumor type, drug type, or geographical factor in the potential benefit of ACEI/ARB drugs (Fig. 3b-e; Table 3).

\section{Second End Point and Safety Outcome}

$E / A$ is the second outcome of this meta-analysis. There were only 3 studies reported $E / A$. A fixed-effects model was performed $\left(p=0.59\right.$ in $Q$ test and $\left.I^{2}=0 \%\right)$. The pooled result shows that ACEI/ARB did not benefit $E / A$ preservation compared to the control group (Fig. 4a; WMD $0.02,95 \%$ CI $[-0.06,0.11], p=0.58)$.

Three studies (228 patients) showed hypotensive events. A total of 3 cases $(2.6 \%)$ of hypotension occurred, all from the experimental group. We conducted a fixed-effects model to evaluate the possibility of ACEI/ARB increasing the risk of hypotension (Fig. $4 \mathrm{~b}, p=0.81$ in $Q$ test, $I^{2}=0 \%$ ). There is no significant difference in the increased risk of hypotension between the experimental group and the control group (risk ratio 3.79, 95\% CI [0.44, 32.89], $p=0.23$ ). ACEI/ARB did not increase the risk of hypotension.

\section{Publication Bias}

Funnel plot for the meta-analysis of the primary outcome is shown in Figure 5. It is not symmetrical on vi- 


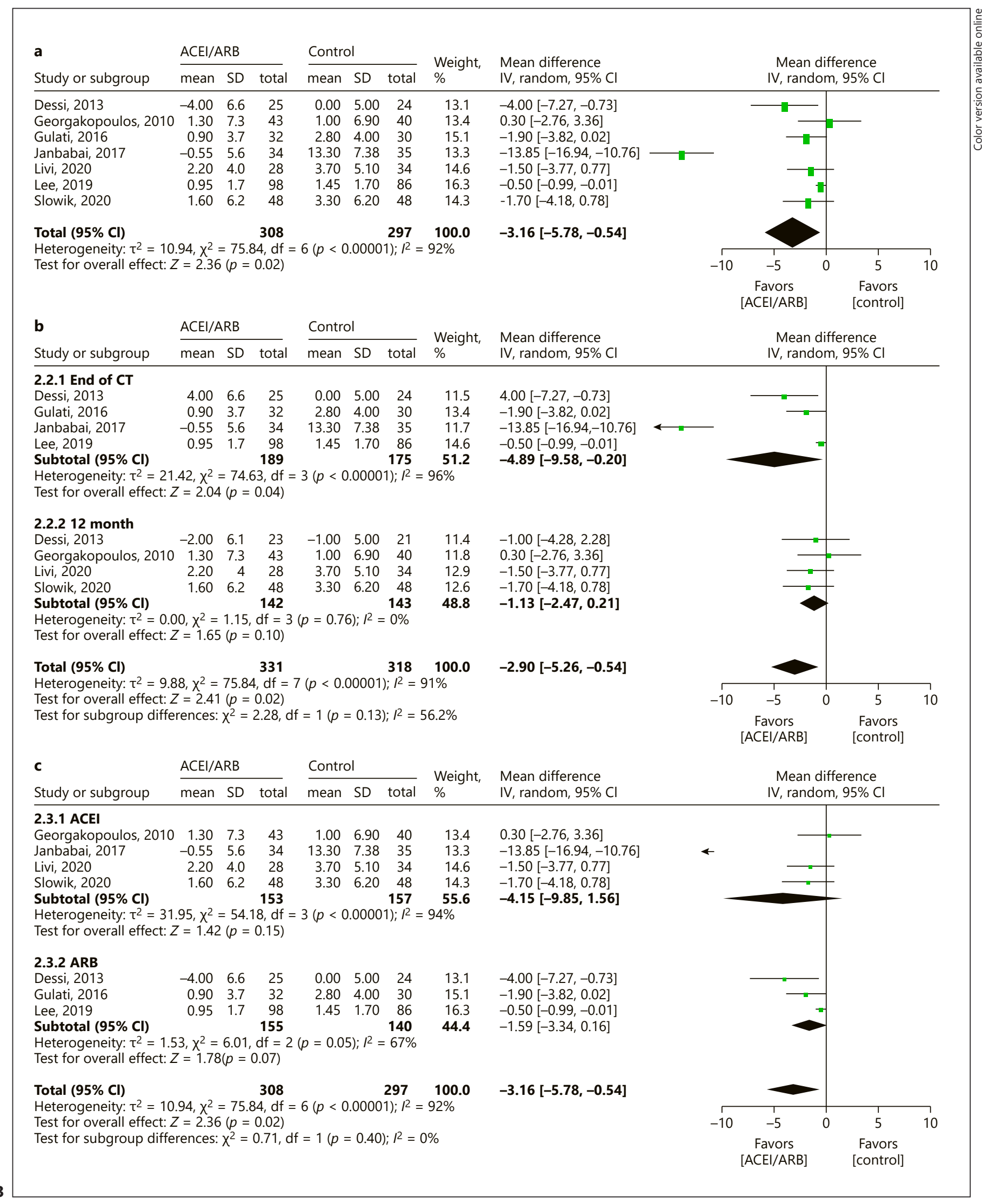

(Figure continued on next page.) 


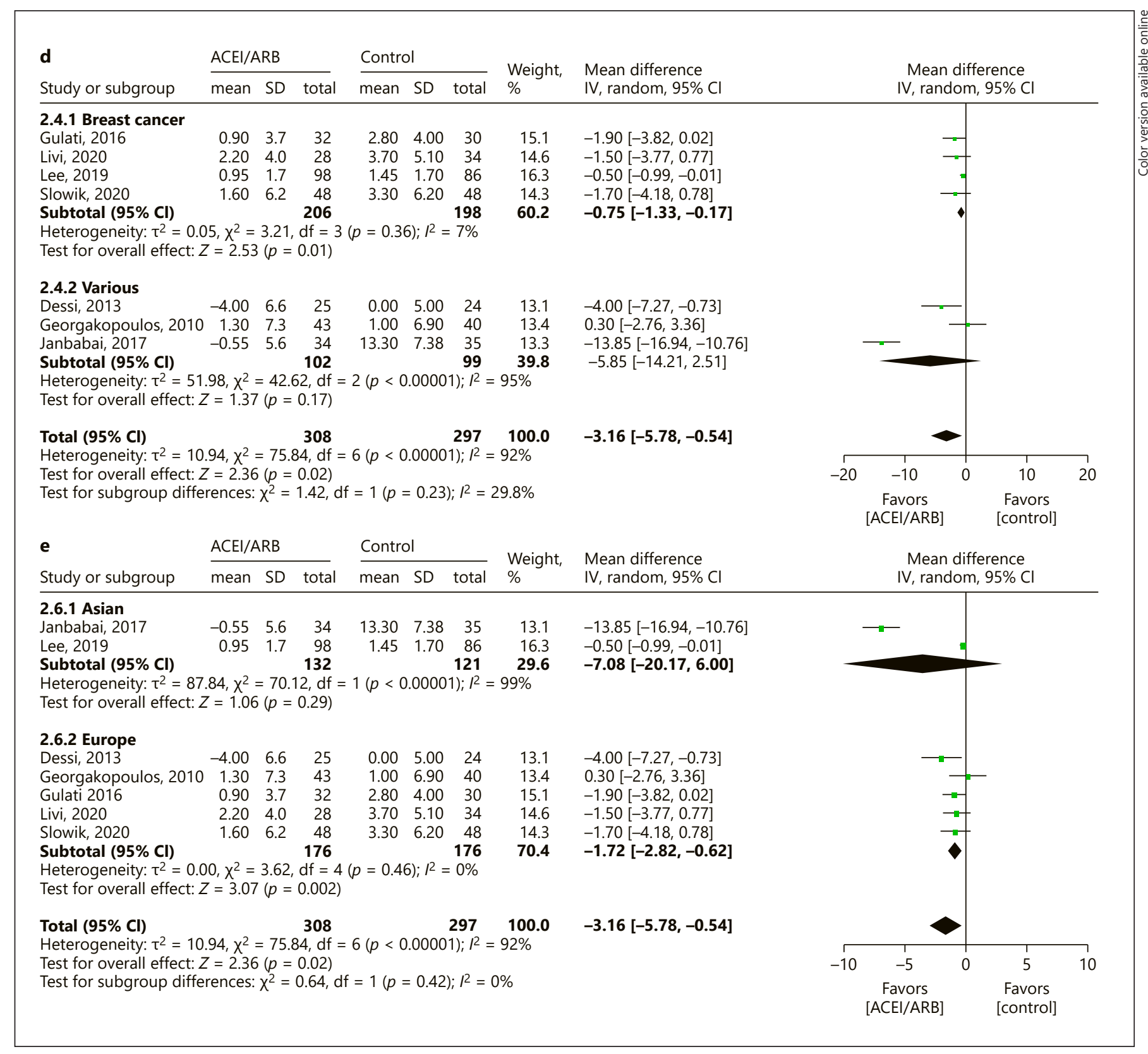

Fig. 3. a Forest plots for the mean difference of LVEF changes between ACEI/ARB and control groups. $\mathbf{b}$ Effect of follow-up time on the mean difference of LVEF changes between ACEI/ARB and control groups. c Effect of drug type on the mean difference of LVEF changes between ACEI/ARB and control groups. $\mathbf{d}$ Effect of cancer type on the mean difference of LVEF changes between

sual inspection, indicating possible publication bias. The meta-analysis of $E / A$ outcome and hypotensive events was difficult to assess publication bias because of the limited studies.

ACEI/ARB Reduces AnthracyclineInduced Cardiotoxicity
ACEI/ARB and control groups. e Effect of geography on the mean difference of LVEF changes between ACEI/ARB and control groups. LVEF, left ventricle ejection fraction; ACEI, angiotensinconverting enzyme inhibitors; $\mathrm{ARB}$, angiotensin-receptor blockers; CI, confidence interval.

\section{Sensitivity Analysis}

Among the 7 included studies, we found an outlier of mean differences on the LVEF change [8], which was considered to be the primary source of substantial hetero- 


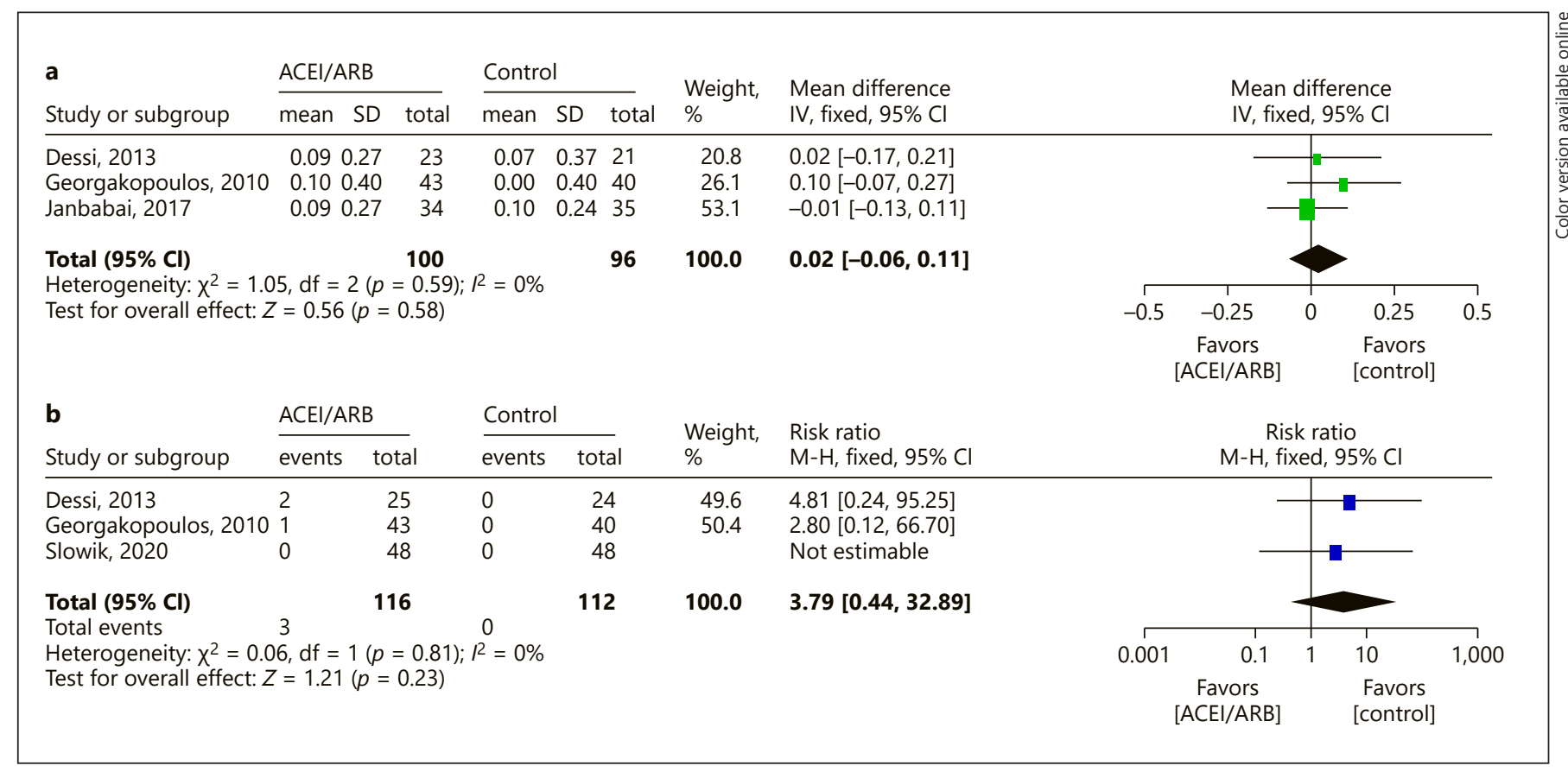

Fig. 4. a Forest plots for the mean difference of $E / A$ changes between ACEI/ARB and control groups. b Forest plots for the risk ratio of hypotension between ACEI/ARB and control groups. ACEI, angiotensin-converting enzyme inhibitors; ARB, angiotensin-receptor blockers; CI, confidence interval; $E / A$, early and late diastolic peak velocity ratio.

Table 3. Subgroup analyses for the effects on mean difference of LVEF changes

\begin{tabular}{|c|c|c|c|c|c|c|}
\hline Subgroup & $\begin{array}{l}\text { Studies, } \\
n\end{array}$ & $\begin{array}{l}\text { Participants, } \\
n\end{array}$ & $\begin{array}{l}\text { WMD } \\
(95 \% \text { CI })\end{array}$ & $\begin{array}{l}p \text { value } \\
\text { for subgroup } \\
\text { effect }\end{array}$ & $I^{2}, \%$ & $\begin{array}{l}p \text { value } \\
\text { for subgroup } \\
\text { difference }\end{array}$ \\
\hline \multicolumn{7}{|l|}{ Follow-up time } \\
\hline EOCT & 4 & 364 & $-4.89(-9.58,-0.20)$ & 0.04 & 96 & \multirow[t]{2}{*}{0.13} \\
\hline 12-months & 4 & 285 & $-1.13(-2.47,0.21)$ & 0.1 & 0 & \\
\hline \multicolumn{7}{|l|}{ Drug type } \\
\hline ACEI & 4 & 310 & $-4.15(-9.85,1.56)$ & 0.15 & 94 & \multirow[t]{2}{*}{0.4} \\
\hline $\mathrm{ARB}$ & 3 & 295 & $-1.59(-3.34,0.16)$ & 0.07 & 67 & \\
\hline \multicolumn{7}{|l|}{ Cancer type } \\
\hline Various & 4 & 201 & $-5.85(-14.21,2.51)$ & 0.17 & 92 & \multirow[t]{2}{*}{0.23} \\
\hline Breast cancer only & 3 & 404 & $-0.75(-1.33,-0.17)$ & 0.01 & 7 & \\
\hline \multicolumn{7}{|l|}{ Geography } \\
\hline Asian & 2 & 253 & $-7.08(-20.17,6.00)$ & 0.29 & 99 & \multirow[t]{2}{*}{0.42} \\
\hline Europe & 5 & 352 & $-1.72[-2.82,-0.62]$ & 0.002 & 0 & \\
\hline
\end{tabular}

EOCT, end of chemotherapy; LVEF, left ventricle ejection fraction; WMD, weighted mean difference; CI, confidence interval.

geneity. After excluding it, the remaining 6 studies showed mild heterogeneity ( $p=0.18$ in $Q$ test, $I^{2}=34 \%$ ). A fixedeffects model was used for meta-analysis in these 6 RCTs, which still indicated that ACEI/ARB was beneficial to the preservation of LVEF (Fig. 6; WMD $-0.70,95 \%$ CI $[-1.15$, $-0.25] p=0.002)$. It further confirms the stability of our meta-analysis. 


\section{Discussion}

Cardiotoxicity is an important side effect of chemotherapy. The common anticancer drugs with cardiotoxicity including anthracyclines, trastuzumab, and PD-1 inhibitors. To our knowledge, this is the first meta-analysis to explore the cardiac protective effect of ACEI/ARB drugs on anthracyclines. Previous meta-analyses $[10,11]$ have discussed the role of ACEI/ARB in chemotherapy, in which most of the included participants used more than one kind of cardiotoxic drugs. Anthracycline is widely used in breast cancer, lymphoma, leukemia, etc., and it is not used in combination with targeted drugs or immune-checkpoint inhibitors in most cases. Learning about necessary measures to prevent anthracycline-induced cardiotoxicity will provide a certain reference for clinical use.

Our meta-analysis suggests that ACEI/ARB has the potential to reduce cardiotoxicity in anthracycline-chemotherapy. ACEI/ARB combined with anthracyclinechemotherapy reduced LVEF by $3.2 \%$ compared with the control group. There was no significant correlation with follow-up time, tumor type, drug type, or geography of intervention. The use of ACEI/ARB did not significantly increase the incidence of hypotension. We planned to evaluate whether ACEI/ARB could reduce the incidence of anthracycline-induced heart failure events. However, there were only 5 heart failure events $(0.8 \%)$ reported in 605 patients (all from Georgakopoulos' study [12]), making meta-analysis impossible. To analyze the benefits of ACEI/ARB on anthracycline-induced heart failure events, more and larger trials are necessary.

Dose-dependent cardiotoxicity is a severe side effect of anthracycline. Swain et al. [13] found that $6.5 \%$ of patients would suffer from cardiac events at a cumulative dose of $150 \mathrm{mg} / \mathrm{m}^{2}$. The cumulative dose of $400 \mathrm{mg} / \mathrm{m}^{2}$ was associated with a $5.1 \%$ risk of heart failure. Georgakopoulos et al. [12] also suggested a $4.8 \%$ incidence of heart failure events at a cumulative dose of $380 \mathrm{mg} / \mathrm{m}^{2}$. Cardiotoxicity caused by anthracycline may present as acute, chronic, and deferred events. Acute cardiotoxicity occurs within a few minutes or a few days of administration [14]. ACEI/ARB may also play a role in acute cardiotoxicity prevention $[15,16]$. Chronic cardiotoxicity usually occurs within 1 year after chemotherapy and deferred cardiotoxicity will occur in following several years after chemotherapy [15]. Our meta-analysis evaluated cardiac function at baseline and 1 year of follow-up, providing a reference for the prevention of anthracycline-induced chronic cardiotoxicity. Patients treated with anthracy-

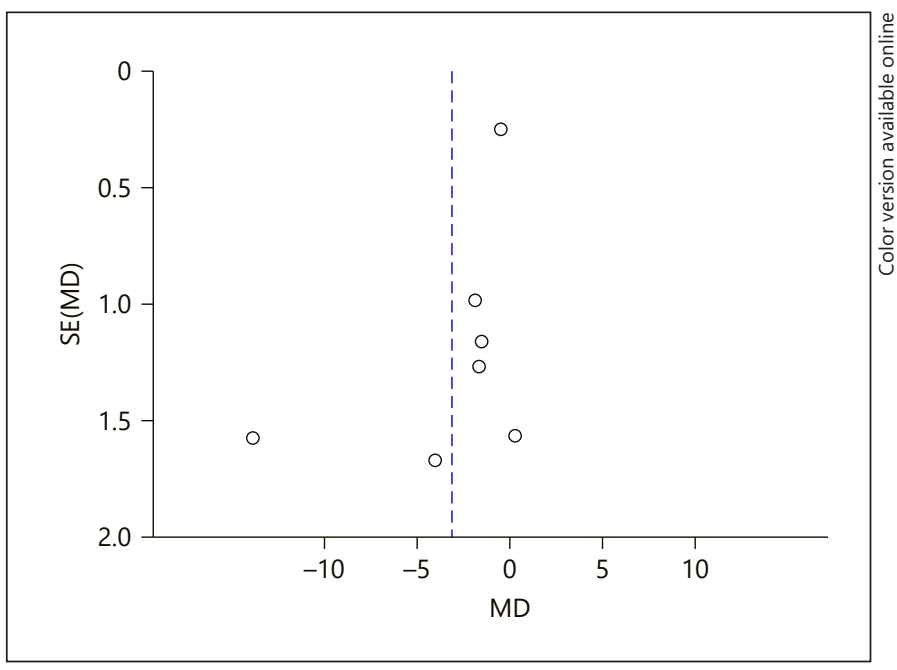

Fig. 5. Funnel plots for the meta-analysis of mean difference of LVEF changes between ACEI/ARB and control groups. LVEF, left ventricle ejection fraction; $\mathrm{ACEI}$, angiotensin-converting enzyme inhibitors; ARB, angiotensin-receptor blockers; CI, confidence interval.

cline will be at a $9 \%$ risk of cardiac toxicity in 5 years [17]. With the progress of treatment and prolonged survival, we need more researches and longer follow-up duration to assess whether ACEI/ARB is beneficial for anthracycline-induced deferred cardiotoxicity. In addition, whether prophylactic use of ACEI/ARB has a difference in the benefits of patients with different cumulative doses should be explored further.

LVEF is a primary indicator of left ventricular function. Left ventricular dysfunction was defined as either a $>10 \%$ decrease in newly occurring LVEF or a $>5 \%$ decrease in LVEF and new LVEF <50\% [17]. Echocardiography and CMR are important tools to measure LVEF. Both 2 tools can only reveal pre-existing heart damage. Anthracycline-induced cardiotoxicity is irreversible, but left ventricular will be possible to recover if intervened before the onset of heart failure symptoms $[18,19]$. Therefore, the discovery of subclinical cardiotoxicity is necessary. Studies have found that imaging by global longitudinal strain from 2D speckle tracking echocardiography can detect myocardial injury earlier [20, 21]. Heck et al. [22] found that obtaining myocardial extracellular volume fraction by late gadolinium enhancement imaging of CMR was helpful in assessing myocardial cells. In addition, N-terminal pro-brain natriuretic peptide and troponin are known to be relatively sensitive indicators of cardiac injury [14,23]. Some studies have used them to assist imaging methods to assess cardiotoxicity $[6,14]$. Of inter- 


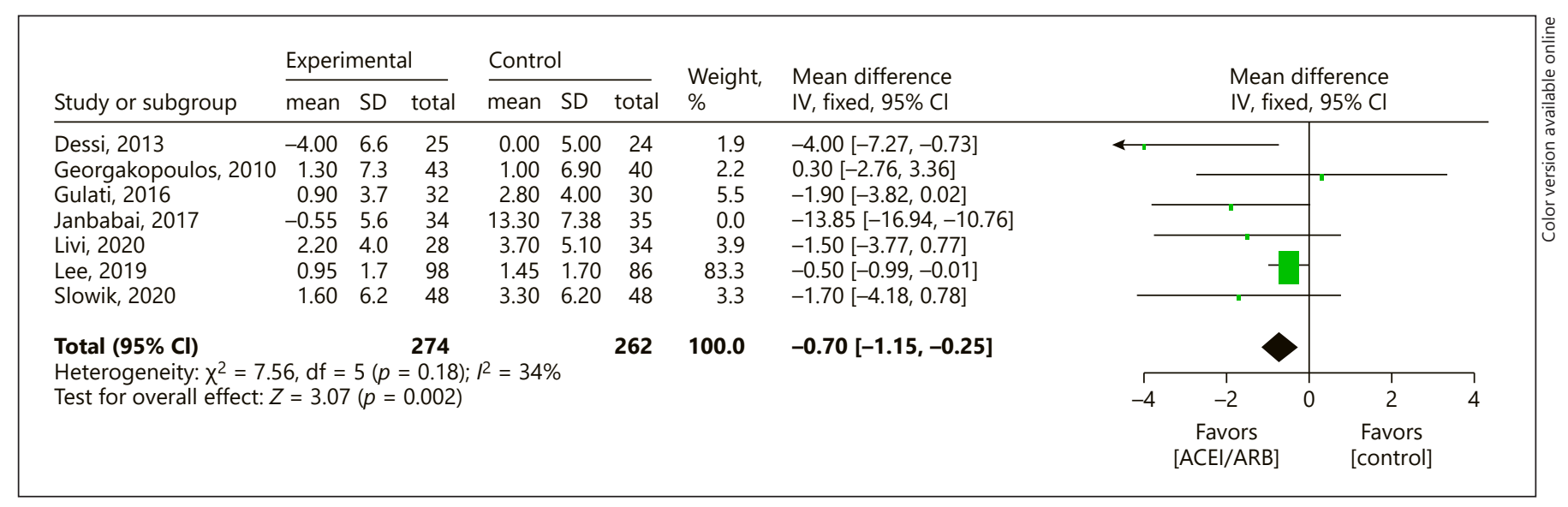

Fig. 6. Forest plots for sensitivity analysis.

est, Rigaud et al. [24] and Gioffre et al. [25] found that specific circulating miRNA may be a specific marker of anthracycline-induced cardiotoxicity.

In addition, Liu et al. [26] found that anthracyclineinduced cardiotoxicity may present as diastolic dysfunction. We performed a meta-analysis on the studies that reported $E / A$ and found no benefit of ACEI/ARB on E/A. The diastolic function of the heart may also be evaluated by combining data such as the left ventricular end-diastolic diameter, the ratio of peak early diastolic trans-mitral velocity to mitral annular velocity $\left(E / e^{\prime}\right)$, and $E$ deceleration time (Dec T). Due to the lack of studies and data, we failed to conduct a meta-analysis of these indicators. More researchers on the changes in diastolic function are needed.

The mechanism of anthracycline-induced myocardial damage includes overactivated oxidative stress; destroy mitochondria; damage of cardiomyocyte DNA; and damage to cellular repair capacity $[27,28]$. ACEIs and ARBs are inhibitors of renin-angiotensin-aldosterone system, which can inhibit the action of angiotensin, promote vasodilation, and lower blood pressure. ACEI/ARB is also an important drug for the treatment of heart failure, which can inhibit ventricular remodeling. Its combination with anthracycline does not affect the efficacy of chemotherapy [29]. Although many studies have suggested the potential of ACEI/ARB to prevent cardiotoxicity induced by anthracycline, few studies have explored the mechanism. In mice models which had doxorubicin-induced cardiomyopathy, enalapril reduced free-radical production and mitochondrial damage and stimulated the $\mathrm{PI} 3 \mathrm{~K} / \mathrm{AKT} / \mathrm{mTOR}$ pathway to protect the myocardium $[30,31]$. The specific mechanism remains to be fur- ther explored. In addition, it is worth noting that Bosch et al. [32] found that the combination of ACEI/ARB and adrenergic beta-receptor blocker also had a benefit in cardiotoxicity prevention, but whether it was more effective than ACEI/ARB alone was unclear.

Our meta-analysis has the following limitations. First, due to the obvious heterogeneity, we can only merge the data using a random-effects model. After the exclusion of an outlier [8], heterogeneity decreased significantly. We initially considered that this outlier was conducted because of the characteristics of participants in the trial. The participants in this trial are required to have an ECOG score of more than 2, suggesting that the enrolled patients had poor physical fitness. Chemotherapy for these cases is more likely to induce cardiotoxicity. The results of our subsequent sensitivity analysis are consistent with the previous results. Second, in three of our included trials [5-7], a small fraction of patients were also treated with trastuzumab. The combination of anthracycline and trastuzumab further increases cardiotoxicity. This may exaggerate the protective effect of ACEI/ARB on the cardiotoxicity induced by anthracycline. Third, the sample sizes of the included studies are relatively small, lacking convincing big data. In some studies, only a conference abstract is provided, and relevant indicators or related cardiac events records are missing. Therefore, the conclusions are not complete, and some conclusions should be cautious. Fourth, selected patients in different RCTs had different characteristics. Some patients had high blood pressure, diabetes, or other high-risk factors for cardiovascular diseases, and these patients may be more sensitive to anthracycline-induced cardiotoxicity. Personal data were not available. We were unable to perform a sub- 
group analysis of cardiovascular risk factors. Finally, the quality of each study is different, and some studies are of relatively low quality, which may confuse the results.

\section{Conclusion}

We found that prophylactic use of ACEI/ARB had a protective effect on the cardiotoxicity of anthracycline, preserving a portion of the decrease in LVEF. More studies and data are imperative to verify our results and determine whether ACEI/ARB can decrease the occurrence of heart failure events induced by anthracycline. A more objective and comprehensive system is needed to assess the effects of anthracycline-induced cardiotoxicity.

\section{Statement of Ethics}

This meta-analysis was performed in accordance with the PRISMA and the Cochrane Handbook guidelines. Our study did not involve individual participation. We did not require Institu- tional Review Board approval or patient informed consent. This study protocol conforms to the provisions of the Helsinki Declaration as revised in 2013.

\section{Conflict of Interest Statement}

The authors declare that they have no conflicts of interest.

\section{Funding Sources}

The authors did not receive any funding.

\section{Author Contributions}

Liming Chen contributed to conception and design. Hong Lin and Guoxi Liang contributed to acquisition of data, analysis, and interpretation of data. All the authors completed, read, and approved the final manuscript.

\section{References}

1 Armenian SH, Lacchetti C, Barac A, Carver J, Constine LS, Denduluri N, et al. Prevention and monitoring of cardiac dysfunction in survivors of adult cancers: American society of clinical oncology clinical practice guideline. J Clin Oncol. 2017;35(8):893-911.

2 Moher D, Liberati A, Tetzlaff J, Altman DG; PRISMA Group. Preferred reporting items for systematic reviews and meta-analyses: the PRISMA statement. PLoS Med. 2009;6(7): e1000097.

3 Higgins J, Green S. Cochrane handbook for systematic reviews of interventions version 5.1.0. London: The Cochrane Collaboration; 2011.

4 Dessi M, Madeddu C, Piras A, Cadeddu C, Antoni G, Mercuro G, et al. Long-term, up to 18 months, protective effects of the angiotensin II receptor blocker telmisartan on Epirubin-induced inflammation and oxidative stress assessed by serial strain rate. Springerplus. 2013;2(1):198.

5 Gulati G, Heck SL, Ree AH, Hoffmann P, Schulz-Menger J, Fagerland MW, et al. Prevention of cardiac dysfunction during adjuvant breast cancer therapy (PRADA): a $2 \times 2$ factorial, randomized, placebo-controlled, double-blind clinical trial of candesartan and metoprolol. Eur Heart J. 2016;37(21):167180.

6 Slowik A, Jagielski P, Potocki P, Streb J, Ochenduszko S, Wysocki P, et al. Anthracycline-induced cardiotoxicity prevention with angiotensin-converting enzyme inhibitor ramipril in low-risk breast cancer women: results of a prospective randomized study. Kardiol Pol. 2020 Feb 25;78(2):131-7.

7 Livi L, Barletta G, Martella F, Desideri I, Scotti V, Becherini C, et al. Pre-specified interim analysis of the SAFE trial (NCT2236806): a 4-arm randomized, double-blind, controlled study evaluating the efficacy and safety of cardiotoxicity prevention in non-metastatic breast cancer patients treated with anthracyclines with or without trastuzumab. Ann Oncol. 2019;30:v72.

8 Janbabai G, Nabati M, Faghihinia M, Azizi S, Borhani S, Yazdani J. Effect of enalapril on preventing anthracycline-induced cardiomyopathy. Cardiovasc Toxicol. 2017;17(2):1309.

9 Lee MH, Chung WB, Song BJ, Park WC, Chae BJ, Youn HJ. The role of candesartan or carvedilol for prevention of anthracycline induced subclinical cardiotoxicity in breast cancer patients. Eur J Heart Fail. 2019;21: 328.

10 Fang K, Zhang Y, Liu W, He C. Effects of angiotensin-converting enzyme inhibitor/angiotensin receptor blocker use on cancer therapy-related cardiac dysfunction: a meta-analysis of randomized controlled trials. Heart Fail Rev. 2020;26(1):101-9.

11 Gujral DM, Lloyd G, Bhattacharyya S. Effect of prophylactic betablocker or ACE inhibitor on cardiac dysfunction \& heart failure during anthracycline chemotherapy \pm trastuzumab. Breast. 2018;37:64-71.
12 Georgakopoulos P, Roussou P, Matsakas E, Karavidas A, Anagnostopoulos N, Marinakis $\mathrm{T}$, et al. Cardioprotective effect of metoprolol and enalapril in doxorubicin-treated lymphoma patients: a prospective, parallel-group, randomized, controlled study with 36-month follow-up. Am J Hematol. 2010;85(11):894-6.

13 Swain SM, Whaley FS, Ewer MS. Congestive heart failure in patients treated with doxorubicin: a retrospective analysis of three trials. Cancer. 2003;97(11):2869-79.

14 Cardinale D, Colombo A, Sandri MT, Lamantia G, Colombo N, Civelli M, et al. Prevention of high-dose chemotherapy-induced cardiotoxicity in high-risk patients by angiotensinconverting enzyme inhibition. Circulation. 2006;114(23):2474-81

15 Nakamae H, Tsumura K, Terada Y, Nakane T, Nakamae M, Ohta K, et al. Notable effects of angiotensin II receptor blocker, valsartan, on acute cardiotoxic changes after standard chemotherapy with cyclophosphamide, doxorubicin, vincristine, and prednisolone. Cancer. 2005; 104(11):2492-8.

16 Piras A, Cadeddu Dessalvi C, Dessi M, Madeddu C, Mercuro G. Preliminary data on a cardioprotective effect of telmisartan in cancer patients treated with epirubicin. Eur Heart J. 2009;30:429.

17 Cardinale D, Colombo A, Bacchiani G, Tedeschi I, Meroni CA, Veglia F, et al. Early detection of anthracycline cardiotoxicity and improvement with heart failure therapy. Circulation. 2015;131(22):1981-8. 
18 Cardinale D, Colombo A, Lamantia G, Colombo N, Civelli M, De Giacomi G, et al. Anthracycline-induced cardiomyopathy: clinical relevance and response to pharmacologic therapy. J Am Coll Cardiol. 2010;55(3):21320.

19 McGowan JV, Chung R, Maulik A, Piotrowska I, Walker JM, Yellon DM. Anthracycline chemotherapy and cardiotoxicity. Cardiovasc Drugs Ther. 2017;31(1):63-75.

20 Negishi T, Thavendiranathan P, Negishi K, Marwick TH. Rationale and design of the strain surveillance of chemotherapy for improving cardiovascular outcomes: the SUCCOUR trial. JACC Cardiovasc Imaging. 2018; 11(8):1098-105.

21 Thavendiranathan P, Poulin F, Lim KD, Plana JC, Woo A, Marwick TH. Use of myocardial strain imaging by echocardiography for the early detection of cardiotoxicity in patients during and after cancer chemotherapy: a systematic review. J Am Coll Cardiol. 2014; 63(25 Pt A):2751-68

22 Heck SL, Gulati G, Hoffmann P, von Knobelsdorff-Brenkenhoff F, Storås TH, Ree AH, et al. Effect of candesartan and metoprolol on myocardial tissue composition during anthracycline treatment: the PRADA trial. Eur Heart J Cardiovasc Imaging. 2018;19(5):54452.
23 Denlinger CS, Sanft T, Baker KS, Broderick G, Demark-Wahnefried W, Friedman DL, et al. Survivorship, version 2.2018, NCCN clinical practice guidelines in oncology. J Natl Compr Canc Netw. 2018;16(10):1216-47.

24 Rigaud VO, Ferreira LR, Ayub-Ferreira SM, Ávila MS, Brandão SM, Cruz FD, et al. Circulating miR-1 as a potential biomarker of doxorubicin-induced cardiotoxicity in breast cancer patients. Oncotarget. 2017;8(4):69947002 .

25 Gioffre S, Chiesa M, Alfieri I, Masson S, Latini R, Sandri MT, et al. Circulating microRNAs as selective markers of anthracyclinesbased therapies in breast cancer patients. Eur Heart J. 2018;39:996.

26 Liu H, Yanqiu L, Fengjuan Y, Hong L, Cuiling L, Rui F, et al. Cardiac diastolic dysfunction may hold promise as markers of cardiotoxicity during daunorubicin treatment in acute nonlymphocytic leukemia patients. Heart. 2013;99:A273.

27 Hahn VS, Lenihan DJ, Ky B. Cancer therapyinduced cardiotoxicity: basic mechanisms and potential cardioprotective therapies. J Am Heart Assoc. 2014;3(2):e000665.

28 Henriksen PA. Anthracycline cardiotoxicity: an update on mechanisms, monitoring and prevention. Heart. 2018;104(12):971-7.
29 Blaes A, Duprez D, Defor T, Shanley R, Beckwith $\mathrm{H}$, Haddad T, et al. Angiotensin Converting Enzyme Inhibitors (ACEI) and doxorubicin pharmacokinetics in women receiving adjuvant breast cancer treatment. Springerplus. 2015;4:32.

30 Hiona A, Lee AS, Nagendran J, Xie X, Connolly AJ, Robbins RC, et al. Pretreatment with angiotensin-converting enzyme inhibitor improves doxorubicin-induced cardiomyopathy via preservation of mitochondrial function. J Thorac Cardiovasc Surg. 2011;142(2): 396-e3.

31 Hullin R, Métrich M, Sarre A, Basquin D, Maillard M, Regamey J, et al. Diverging effects of enalapril or eplerenone in primary prevention against doxorubicin-induced cardiotoxicity. Cardiovasc Res. 2018;114(2):272-81.

32 Bosch X, Rovira M, Sitges M, Domènech A, Ortiz-Pérez JT, de Caralt TM, et al. Enalapril and carvedilol for preventing chemotherapyinduced left ventricular systolic dysfunction in patients with malignant hemopathies: the OVERCOME trial (preventiOn of left Ventricular dysfunction with Enalapril and caRvedilol in patients submitted to intensive ChemOtherapy for the treatment of Malignant hEmopathies). J Am Coll Cardiol. 2013; 61(23):2355-62. 